\title{
Extracellular vesicles in cardiovascular diseases
}

Shihui Fu ${ }^{1,2}$, Yujie Zhang ${ }^{3}$, Yulong Li', Leiming Luo ${ }^{1}$, Yali Zhao ${ }^{4}$ and Yao Yao, ${ }^{5,6}$

\begin{abstract}
Due to the continued high incidence and mortality rate worldwide, there is still a need to develop new strategies for the prevention, diagnosis and treatment of cardiovascular diseases (CVDs). Proper cardiovascular function depends on the coordinated interplay and communication between cardiomyocytes and noncardiomyocytes. Extracellular vesicles (EVs) are enclosed in a lipid bilayer and represent a significant mechanism for intracellular communication. By containing and transporting various bioactive molecules, such as micro-ribonucleic acids (miRs) and proteins, to target cells, EVs impart favourable, neutral or detrimental effects on recipient cells, such as modulating gene expression, influencing cell phenotype, affecting molecular pathways and mediating biological behaviours. EVs can be released by cardiovascular system-related cells, such as cardiomyocytes, endotheliocytes, fibroblasts, platelets, smooth muscle cells, leucocytes, monocytes and macrophages. EVs containing miRs and proteins regulate a multitude of diverse functions in target cells, maintaining cardiovascular balance and health or inducing pathological changes in CVDs. On the one hand, miRs and proteins transferred by EVs play biological roles in maintaining normal cardiac structure and function under physiological conditions. On the other hand, EVs change the composition of their miR and protein cargoes under pathological conditions, which gives rise to the development of CVDs. Therefore, EVs hold tremendous potential to prevent, diagnose and treat CVDs. The current article reviews the specific functions of EVs in different CVDs.
\end{abstract}

\section{FACTS}

- By containing and transporting various bioactive molecules, extracellular vesicles represent a significant mechanism for intracellular communication and impart favourable, neutral or detrimental effects on recipient cells, such as modulating gene expression, influencing cell phenotype, affecting molecular pathways and mediating biological behaviours.

- Extracellular vesicles play significant roles in maintaining normal cardiac structure and

Correspondence: Leiming Luo (luoleiming@vip.sina.com) or Yali Zhao (zhaoyl301@163.com) or Yao Yao (yaoyao@nsd.pku.edu.cn)

'Department of Geriatric Cardiology, Chinese People's Liberation Army General Hospital, Beijing 100853, China

2Department of Cardiology, Hainan Hospital of Chinese People's Liberation Army General Hospital, Sanya 572013, China

Full list of author information is available at the end of the article These authors contributed equally: Shihui Fu, Yujie Zhang, Yulong Li Edited by M. V. Niklison Chirou function under physiological conditions and change their composition under pathological conditions to promote the development of cardiovascular diseases.

- Cardiovascular diseases are the leading cause of death, accounting for almost a third of deaths worldwide. Extracellular vesicles play fundamental roles in regulating physiological and pathological functions and hold tremendous potential to monitor and treat cardiovascular diseases.

\section{Open questions}

- There is still a need to develop new strategies for the prevention and treatment of different cardiovascular diseases, given their continued high incidence and mortality rate worldwide.

- Little is known about extracellular vesicle-mediated regulation of cardiomyocytes and noncardiomyocytes within the healthy and diseased heart. 
- There is a lack of broad and detailed understanding of extracellular vesicle functions and a need for the development of new and personalized extracellular vesicle-based therapies to treat different cardiovascular diseases.

\section{Introduction}

Cardiovascular diseases (CVDs) are the leading cause of death, accounting for almost a third of deaths worldwide ${ }^{1}$. Although advances in cardiovascular research and care have increased patient survival, there is still a need to develop new strategies for CVD prevention and treatment, given the continued high incidence and mortality rate in many countries ${ }^{2}$. Extracellular vesicles (EVs) are enclosed in a lipid bilayer and represent a by-product released by cells (Fig. 1$)^{3}$. In addition to direct cell-cell contact or the transport of secreted molecules, EVs also participate in intercellular communication ${ }^{4}$. By containing and transporting various bioactive molecules, such as proteins, lipids, messenger ribonucleic acids (mRNAs), micro-ribonucleic acids (microRNAs, miRs) and deoxyribonucleic acids (DNAs), to target cells, EVs impart favourable, neutral or detrimental effects on recipient cells, such as modulating gene expression, influencing cell phenotype, affecting molecular pathways and mediating biological behaviours ${ }^{5}$.

EVs encompass three subtypes, exosomes, microvesicles and apoptotic bodies, which have different routes of intracellular formation, sizes and contents ${ }^{6}$. Whereas exosomes are released continuously from cells, microvesicles and apoptotic bodies are predominantly released from activated or apoptotic cells. Exosomes are released from cells via the endolysosomal pathway, and microvesicles and apoptotic bodies are formed by budding from the plasma membrane. Apoptotic bodies (>1000 nm) form by apoptotic cell membrane blebbing to recycle contents and contain cell debris, genomic DNA (gDNA) and proteins. Microvesicles $(150-1000 \mathrm{~nm})$ form through outward pinching off of the plasma membrane and contain RNAs, proteins and lipids. Exosomes, the smallest type of EV (30-150 nm), originate within multivesicular bodies and carry RNAs, proteins and lipids. The plasma membrane buds inward, fills with cytoplasmic contents and retains plasma membrane proteins specific to the cell of origin. Early endosomes are formed and then mature into late endosomes. This plasma membrane further buds inward and forms intraluminal vesicles with the aid of endosomal sorting complex required for transport. Once filled with these exosomes, late endosomes become multivesicular bodies, which might either fuse with the plasma membrane and secrete exosomes or deliver their contents to the lysosome for degradation. Molecular mechanisms controlling exosome production are far from fully understood, and some of these mechanisms appear to vary between cell types.

Several studies have recently indicated that EVs can be released by cardiovascular system-related cells, such as cardiomyocytes, endotheliocytes, fibroblasts, platelets, smooth muscle cells (SMCs), leucocytes, monocytes and macrophages (Fig. 2) ${ }^{7}$. On the one hand, EVs play biological roles in maintaining normal cardiac structure and function under physiological conditions. On the other hand, EVs change their composition under pathological conditions and contribute to the development of CVDs ${ }^{8}$. Therefore, EVs hold tremendous potential to monitor and treat CVDs. Increasing evidence suggests that the effects

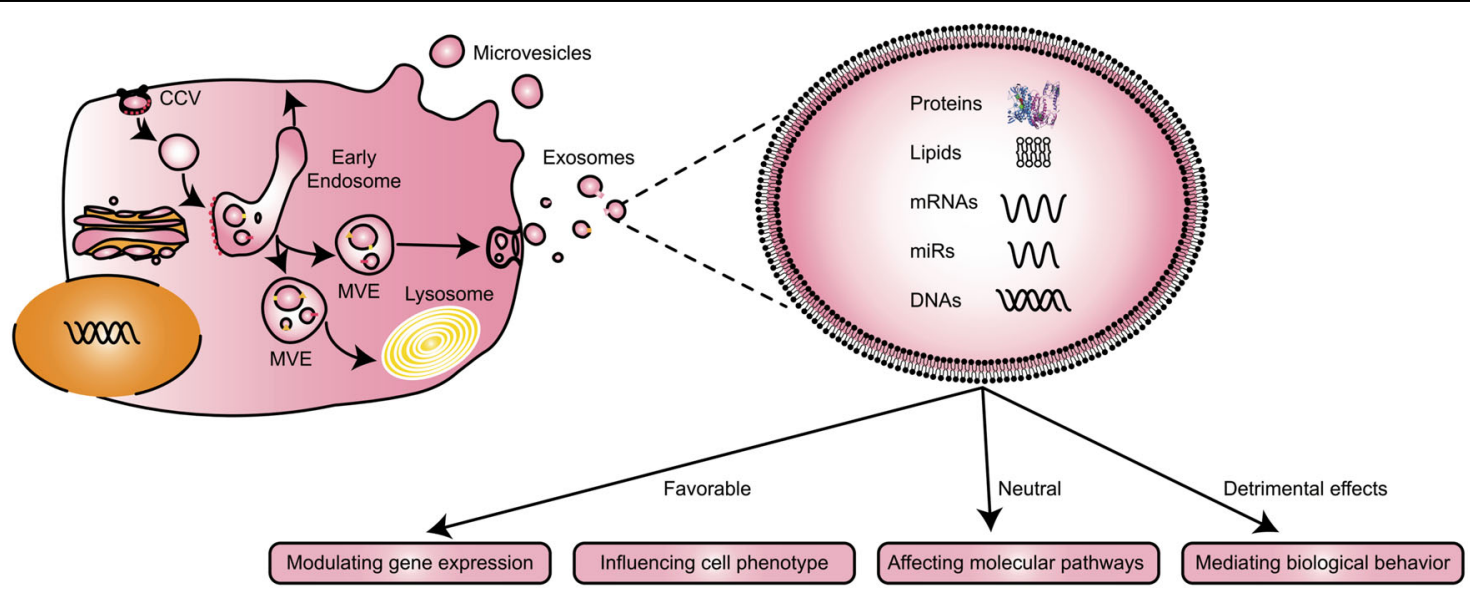

Fig. 1 Origins, contents and roles of extracellular vesicles. Extracellular vesicles (EVs) are enclosed in a lipid bilayer and represent a by-product released by cells. In addition to direct cell-cell contact or the transport of secreted molecules, EVs also participate in intercellular communication. By containing and transporting various bioactive molecules, such as proteins, lipids, messenger ribonucleic acids (mRNAs), micro-ribonucleic acids (microRNAs, miRs) and deoxyribonucleic acids (DNAs), to target cells, EVs impart favourable, neutral or detrimental effects on recipient cells, such as modulating gene expression, influencing cell phenotype, affecting molecular pathways and mediating biological behaviours. 


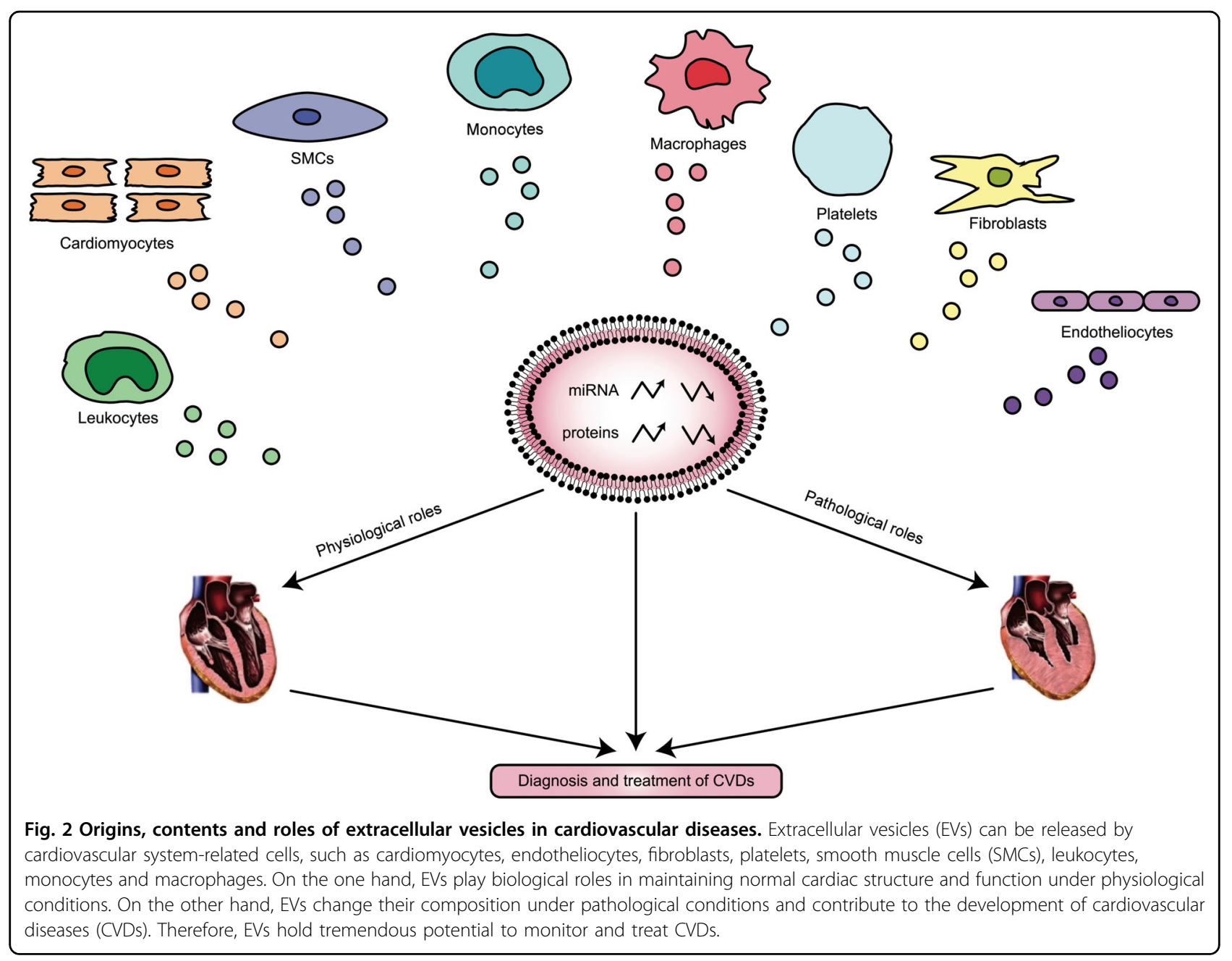

Table 1 Extracellular vesicle-based miRs and targets in cardiovascular diseases.

\begin{tabular}{|c|c|c|}
\hline Cardiovascular diseases & MiRs & Targets \\
\hline $\begin{array}{l}\text { Myocardial ischaemia } \\
\text { and injury }\end{array}$ & 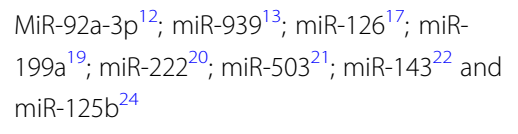 & $\begin{array}{l}\text { Mitochondrially encoded cytochrome c oxidase } \mathrm{I}^{15} \text {; stromal cell-derived } \\
\text { factor- } 1^{18} \text {; intercellular adhesion molecule } 1^{20} \text {; ephrin-B2 } 2^{21} \text {; vascular } \\
\text { endothelial growth factor }{ }^{21} \text { and Kruppel-like factor } 2^{22}\end{array}$ \\
\hline Atrial fibrillation & MiR-199a-3p $p^{29} ; \operatorname{miR}-21-3 p^{31}$ & Gata-binding $4^{29}$ \\
\hline $\begin{array}{l}\text { Cardiac hypertrophyand } \\
\text { failure }\end{array}$ & $\begin{array}{l}\text { MiR-21-5 } p^{30} ; \operatorname{miR}-21-3 p^{31} ; \operatorname{miR}-320^{33} \text { and } \\
\text { miR-146a }\end{array}$ & $\begin{array}{l}\text { Sorbin and SH3 domain containing protein } 2^{31} \text {; signal transducer and } \\
\text { activator of transcription } 3^{32} \text { and small ubiquitin-like proteins modifier } 1^{35}\end{array}$ \\
\hline
\end{tabular}

MiR micro-ribonucleic acid.

of EVs on target cells are mainly dependent on miRs and proteins transferred by EVs ${ }^{9}$. Depending on the condition of the source cells, EVs containing miRs and proteins have been shown to regulate a multitude of diverse functions in target cells, maintaining cardiovascular balance and health or inducing pathological changes in CVDs (Table 1). The current article reviews the specific functions of EVs in different CVDs ${ }^{10}$.

\section{Myocardial ischaemia and injury}

EVs and their miRs are key players in mediating inflammatory and coagulative reactions involving endotheliocytes, platelets, SMCs and inflammatory cells and thus contribute to the development of atherosclerosis and angiogenesis ${ }^{11}$. Endotheliocytes and platelets have been shown to be the major sources of EVs in patients with stable coronary artery disease (CAD). Atherosclerotic 
conditions promote the packaging of miR-92a-3p into endothelial EVs, which regulates angiogenesis in recipient endotheliocytes by a thrombospondin 1-dependent mechanism ${ }^{12}$. Coronary serum EVs isolated from patients with myocardial ischaemia promote angiogenesis via the miR-939-mediated nitric oxide signalling pathway ${ }^{13}$. EVs derived from activated endotheliocytes and inflammatory cells, such as monocytes, and especially those expressing cell adhesion and procoagulant molecules, indicate early vascular dysfunction ${ }^{14}$. Low mitochondrial encoded cytochrome c oxidase I (MT-COI) in monocyte-specific EVs from stable CAD patients is used to identify a population that is at risk for new cardiovascular events ${ }^{15}$. EVs from macrophage-derived foam cells can promote SMC migration and adhesion, which might be mediated by the integration of EVs into the SMCs and the subsequent activation of extracellular regulated protein kinase $(\mathrm{ERK})$ and protein kinase $\mathrm{B}(\mathrm{PKB})^{16}$. Endothelial EV-mediated transfer of miR-126 promotes the repair of endotheliocyte damage caused by hyperglycaemic conditions. Intravascular injection of endothelial EVs containing miR-126 accelerated reendothelialization after electric denudation of the endothelium in vivo ${ }^{17}$. Moreover, endotheliocytes shed EVs containing miR-126 during atherosclerosis and induce the production of stromal cellderived factor-1 (SDF-1) in recipient vascular cells. SDF-1 counteracts endotheliocyte apoptosis, recruits progenitor cells and improves plaque stability ${ }^{18}$. Increased expression of miR-126 and miR-199a in EVs but not plasma has been associated with reduced major adverse cardiovascular events in patients with stable $\mathrm{CAD}^{19}$.

Endotheliocytes with increased expression of intercellular adhesion molecule 1 (ICAM-1), an adhesion molecule, in the cell membrane show increased endothelial-monocyte adhesion in cell culture, and endothelial EVs play anti-inflammatory roles by reducing endothelial ICAM-1 expression through transferring functional miR-222 into recipient cells ${ }^{20}$. Hyperglycaemiaactivated miR-503 is packaged into endothelial EVs and delivered to vascular pericytes, resulting in reduced expression of Ephrin-B2 (EFNB2) and vascular endothelial growth factor (VEGF) in these cells, followed by impaired migration and proliferation ${ }^{21}$. Shear stress and Kruppellike factor 2 (KLF2), a shear-responsive transcription factor, cause miR-143 upregulation in endotheliocytes and control the phenotypes of SMCs. EVs derived from KLF2expressing endotheliocytes reduce atherosclerotic lesion formation, improve atherosclerotic plaque stability and promote progenitor cell incorporation in the aorta in $\mathrm{ApoE}^{-1-}$ mice $^{22}$. Mesenchymal stromal cell (MSC)derived EVs attenuate myocardial ischaemia-reperfusion injury in mice by shuttling miR-182, which modifies the polarisation status of macrophages ${ }^{23}$. Hypoxia-induced MSC-derived EVs facilitate ischaemic cardiac repair through miR-125b-mediated amelioration of cardiomyocyte apoptosis and prevention of cardiomyocyte death in myocardial infarction $(\mathrm{MI})^{24}$.

Cardiovascular risk factors change the content of plasma EVs, and EVs play a crucial role in intercellular signalling, influencing the development of myocardial injury $^{25}$. Due to increased release from activated or apoptotic cells, EVs are increased in myocardial injury and represent biomarkers for $\mathrm{MI}^{26}$. In MI, cardiomyocytes secrete EVs enriched in miRs related to endothelial proliferation and differentiation, including miR-143 and miR222 , as a response to hypoxic conditions and inflammation ${ }^{27}$. A circulating EV protein network that is also involved in the inflammatory response has been found to be associated with $\mathrm{MI}^{28}$. Moreover, EVs play a fundamental role in the reorganisation of heart muscle after MI, suggesting an enormous potential for interventions by increasing or decreasing miR-containing EVs.

\section{Atrial enlargement and fibrillation}

An enlarged left atrium is closely related to atrial fibrillation, and patients with atrial fibrillation develop cardiac tissue thickening or fibrosis. In patients with atrial fibrillation, miR-21-3p has been found in EVs and plasma and associated with abnormal enlargement of cardiac tissue. MiR-199a-3p in extracellular matrix-derived EVs has been shown to promote cardiac tissue growth and affect atrial electrical function by repressing homeodomain-only protein X (HOPX) expression and increasing Gata-binding 4 (Gata4) acetylation ${ }^{29}$.

\section{Cardiac hypertrophy and failure}

EVs have been shown to mediate cross-talk between cardiomyocytes and fibroblasts in cardiac hypertrophy and have become disease biomarkers and diagnostic tools for cardiac hypertrophy and heart failure (HF). Previous studies have shown a significant role of miRs and proteins transferred by EVs in the development of HF and protection against undesired modifications to heart muscle. MiR-21-5p contributes to EV-mediated heart repair by enhancing angiogenesis and cardiomyocyte survival through the phosphatase and tensin homologue (PTEN)/ PKB pathway. However, the pathological conditions of HF patients impair their regenerative activities by dysregulating $\mathrm{miR}-21-5 \mathrm{p}$ in cardiac-derived $\mathrm{EVs}^{30}$. Cardiac fibroblast-derived EVs enriched in miR-21-3p serve as paracrine signalling-mediators of cardiomyocyte hypertrophy. MiR-21-3p induces cardiomyocyte hypertrophy by targeting sorbin and $\mathrm{SH} 3$ domain containing protein 2 (SORBS2). Furthermore, pharmacological inhibition of miR-21-3p reduces the development of cardiac hypertrophy in an Ang II-induced mouse model ${ }^{31}$. EVs secreted from hypertrophied cardiomyocytes contain heat shock protein 90 (HSP90) and interleukin (IL-6) to maintain the 
signal transducer and activator of transcription 3 (STAT3) pathway signalling ${ }^{32}$. EVs released from diabetic cardiomyocytes inhibit the proliferation, migration, and tube formation of endotheliocytes through a mechanism involving the transfer of EVs containing miR-320 33 . MiR146a-loaded EVs shed by endotheliocytes are messengers in the development of peripartum cardiomyopathy and biomarkers for the diagnosis of peripartum cardiomyopathy $^{34}$. A $16 \mathrm{kDa}$-terminal prolactin fragment $(16 \mathrm{~K}$ PRL) induces increased miR-146a expression in endotheliocytes and promotes the release of miR-146a-loaded EVs. MiR-146a suppresses small ubiquitin-like protein modifier 1 (SUMO1) expression and induces cardiac dysfunction in maladaptive hypertrophy ${ }^{35}$.

\section{CVD prevention and treatment}

$\mathrm{MiR}$ and protein contents have been shown to differ in the EVs from lean and fatty rats ${ }^{36}$. EVs released from hypertrophic adipocytes impair endotheliocyte function, suggesting a possible role for EVs in obesity-related atherosclerosis ${ }^{37}$. Adipocyte-derived EVs have been shown to promote macrophage activation by delivering adipocyte-dominant transcripts (mRNAs and miRs) to macrophages ${ }^{38}$. Adipocyte-derived EVs might inhibit the regulation of macrophage cholesterol efflux and promote the development of atherosclerosis ${ }^{39}$. Adipocyte-derived EVs promote plaque burden and exacerbate vulnerable atherosclerosis by inducing angiogenesis of the vasa vasorum in diabetic $\mathrm{ApoE}^{-1-}$ mice $^{40}$. MiR-93-5penhanced adipose-derived stromal cell-derived EVs prevent cardiac injury by inhibiting autophagy and inflammation and attenuate acute MI-induced myocardial damage $^{41}$. Visceral fat-derived EVs, regardless of obesity, facilitate macrophage-derived foam cell formation through the prevention of adenosine triphosphate (ATP)binding cassette transporter 1 (ABCA1)- and ATPbinding cassette subfamily G member 1 (ABCG1)-mediated cholesterol efflux ${ }^{42}$. Only obese visceral fat-derived EVs are capable of inducing macrophage polarisation. EVs from the abdominal adipose tissue of obese mice but not lean mice activate monocyte differentiation into macrophages and induce insulin resistance ${ }^{43}$. The lipid modifying enzyme phospholipase D2 (PLD2) has been demonstrated to be involved in EV production and acts downstream of the small guanosine triphosphatase (GTPase) adenosine diphosphate (ADP)-ribosylation factor 6 (ARF6). PLD2 might integrate a significant number of signalling pathways that participate in EV release. Long-term exercise-derived circulating EVs protect the heart from myocardial ischaemia/reperfusion injury via exosomal miR-342-5p ${ }^{44}$. Long-term exercise training potentially enhances silent information regulator 1 (SIRT1) signalling, attenuates cardiac inflammation, and provides cardioprotection in D-galactose-induced ageing rats $^{45}$. Cigarette smoke-induced EVs promote thrombin generation in normal human plasma and contribute to increased cardiovascular risk in smokers ${ }^{46}$.

$\mathrm{MiR}$ and protein profiles have been shown to differ in EVs from hypertensive rats compared with normotensive rats ${ }^{47}$. Human embryonic kidney 293 cells containing SV40 large T antigen (HEK293T cells) overexpressing angiotensin II type 1 receptor (AT1R) secreted the EVs enriched in AT1Rs when subjected to osmotic stretch in vitro ${ }^{48}$. Likewise, in mice subjected to transverse aortic constriction to induce pressure overload, EVs were released into the serum that had a 100-fold increase in AT1R density. These AT1R-enriched EVs transferred functional AT1R to recipient wild-type HEK293T cells in vitro and to heart muscle in AT1R-knockout mice in vivo. There have been obvious increases in EVs in the plasma of diabetic individuals, and diabetic conditions render EVs ineffective in activating survival pathways in cardiomyocytes $^{49}$. Insulin resistance increases EV secretion, and EVs might modulate insulin signalling because EVs are preferentially internalised by leucocytes and alter leucocyte function ${ }^{50}$. In the absence of diabetes mellitus, cardioprotective EVs have been shown to activate the tolllike receptor 4 (TLR-4)-extracellular signal-regulated kinase 1 and 2 (ERK1/2) pathway. Exosomal mammalian sterile 20-like kinase 1 (Mst1)-enriched EVs released from cardiac microvascular endothelial cells to cardiomyocytes worsen diabetic cardiomyopathy by inhibiting cardiomyocyte autophagy, promoting cardiomyocyte apoptosis and suppressing glucose metabolism ${ }^{51}$.

Additional investigations have explored the use of EVs as a delivery vehicle for various therapies because of their ability to easily cross biological membranes (Table 2$)^{52-54}$. An engineered hydrogel patch capable of slowly releasing and providing sustained delivery of EVs secreted from induced pluripotent stem cell (iPSC)-derived cardiomyocytes has been developed to promote ejection fraction recovery, reduce arrhythmic burden, prevent cardiomyocyte apoptosis, reduce infarct size and inhibit cell hypertrophy when implanted onto rat hearts after $\mathrm{MI}^{55}$. In a preclinical MI mouse model, localised injection of miR21-loaded EVs effectively delivered miR-21 into cardiomyocytes and endotheliocytes, drastically inhibited cell apoptosis and significantly restored cardiac function ${ }^{56}$. EVs engineered by ischaemic myocardium-targeting peptides can specifically target the ischaemic myocardium, and MSC-derived ischaemic myocardiumtargeting peptide-containing EVs exert enhanced therapeutic effects on $\mathrm{MI}^{57}$.

\section{Stem cell-derived EVs}

EVs mimic the cardioprotective properties of various stem cells (Fig. 3), including MSCs, haematopoietic stem cells (HSCs) and iPSCs ${ }^{58-60}$. These EVs modulate 
Table 2 Extracellular vesicle-based therapeutic options in cardiovascular diseases.

\begin{tabular}{|c|c|}
\hline Therapies & Roles \\
\hline Intravascular injection of endothelial EVs containing miR-126 ${ }^{17}$ & $\begin{array}{l}\text { Accelerating reendothelialization after electric denudation of the } \\
\text { endothelium }{ }^{17}\end{array}$ \\
\hline MSCs-derived EVs ${ }^{23}$ & $\begin{array}{l}\text { Modifying the polarisation status of macrophages and attenuating myocardial } \\
\text { ischaemia-reperfusion injury }\end{array}$ \\
\hline Hypoxia-induced MSC-derived EVs ${ }^{24}$ & $\begin{array}{l}\text { Ameliorating cardiomyocyte apoptosis and preventing cardiomyocyte death in } \\
\mathrm{Ml}^{24}\end{array}$ \\
\hline cTnl-targeted EVs carrying miR-590-3p from MSCs ${ }^{70}$ & $\begin{array}{l}\text { Promoting cardiomyocyte proliferation and restoring cardiac function in the } \\
\text { peri-Ml area }{ }^{70}\end{array}$ \\
\hline $\begin{array}{l}\text { An engineered hydrogel patch capable of slowly releasing and providing } \\
\text { sustained delivery of EVs secreted from iPSC-derived cardiomyocytes }{ }^{55}\end{array}$ & $\begin{array}{l}\text { Promoting ejection-fraction recovery, lowering arrhythmic burden, reducing } \\
\text { infarct size and inhibiting cell hypertrophy after } \mathrm{Ml}^{55}\end{array}$ \\
\hline Cell-free delivery of EVs secreted from iPSC-derived cardiomyocytes ${ }^{71}$ & Promoting heart recovery in myocardial injury ${ }^{71}$ \\
\hline Localised injection of miR-21-loaded EVs ${ }^{56}$ & Inhibiting cell apoptosis and restoring cardiac function in preclinical $\mathrm{MI}^{56}$ \\
\hline EVs engineered by ischaemic myocardium-targeting peptides ${ }^{57}$ & Enhancing therapeutic effects on $\mathrm{Ml}^{57}$ \\
\hline
\end{tabular}

EV extracellular vesicle, MiR micro-ribonucleic acid, MSCs mesenchymal stromal cells, MI myocardial infarction, iPSCs induced pluripotent stem cells.

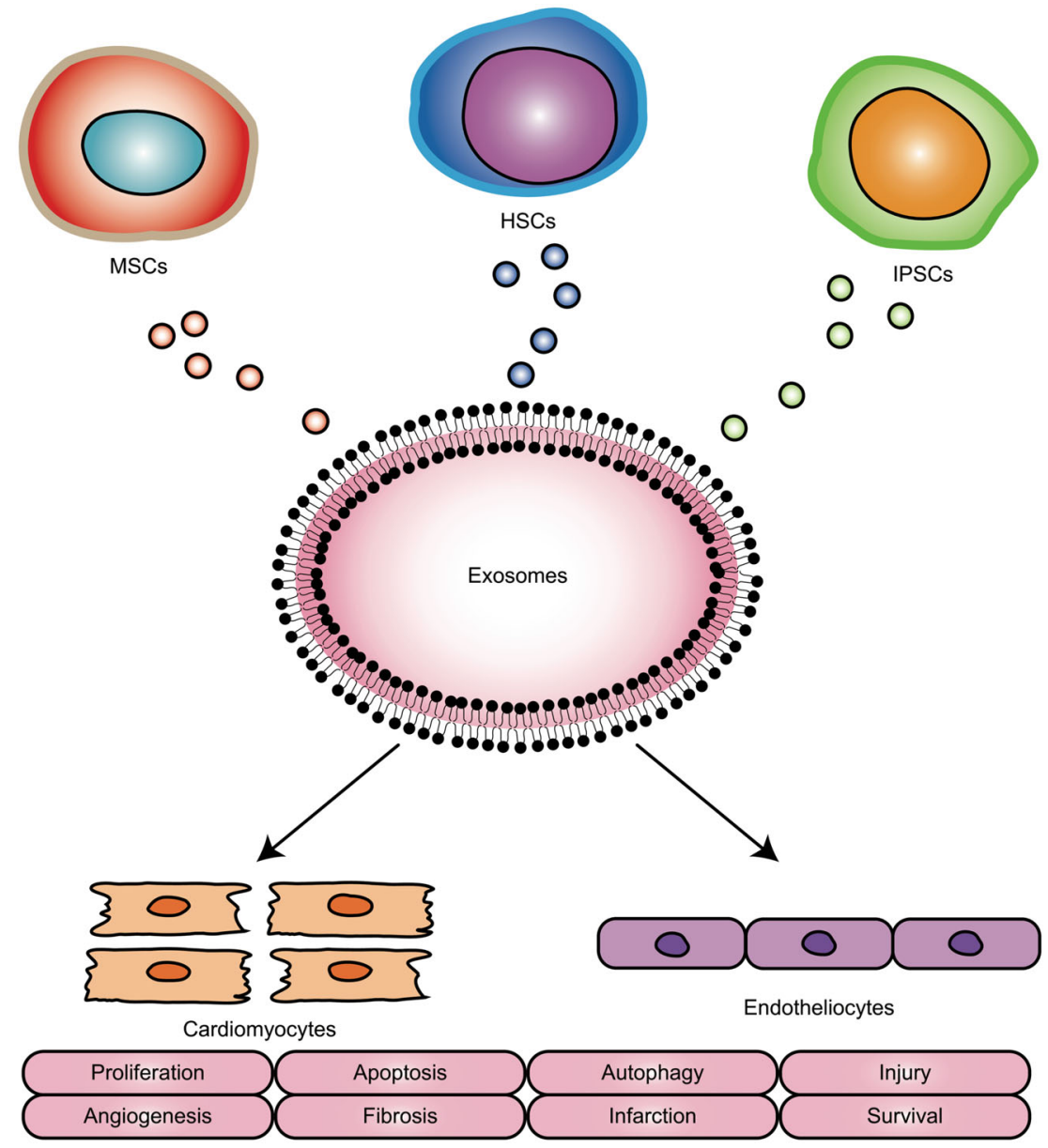

Fig. 3 Origins and roles of stem cell-derived extracellular vesicles. Extracellular vesicles (EVs) mimic the cardioprotective properties of various stem cells, including mesenchymal stromal cells (MSCs), haematopoietic stem cells (HSCs) and induced pluripotent stem cell (iPSCs). These EVs modulate cardiomyocytes and endotheliocytes by stimulating cell proliferation, decreasing cell apoptosis, inhibiting cell autophagy, promoting angiogenesis, reducing tissue fibrosis, preventing cardiovascular injury, treating myocardial infarction and improving cardiac survival.

cardiomyocytes and endotheliocytes by stimulating cell proliferation, decreasing cell apoptosis, inhibiting cell autophagy, promoting angiogenesis, reducing tissue fibrosis, preventing cardiovascular injury, treating MI and improving cardiac survival. Several studies examining the roles of MSC-secreted EVs have found that these EVs are 
involved in cardioprotective paracrine effects ${ }^{61}$. MSCs originate in the bone marrow, and MSC-derived EVs influence both endotheliocytes and cardiomyocytes. MSC-derived EVs affect endotheliocytes and cardiomyocytes by stimulating angiogenesis and decreasing apoptosis by preserving mitochondrial membrane potential through miR-19a enrichment ${ }^{62}$. MSC-derived EVs can protect cardiac tissue from ischaemic injury by promoting blood vessel formation, reducing infarct size and preserving cardiac function ${ }^{63}$. MSCs have been shown to mediate cardioprotective paracrine effects by secreting EVs ${ }^{64}$. MSC-EVs significantly improve cardiac survival, enhance capillary density, reduce cardiac fibrosis and restore longterm function, suggesting that EVs released from MSCs, can act as shuttles and stimulate the proliferation and migration of cardiomyocytes ${ }^{65}$. Whereas EVs harvested from stem cells in vitro might protect against cardiac injury and promote cardiac repair, EVs secreted from endogenous cardiac cells might play a more deleterious role in the progression of CVDs. Circulating myocardial miRs from infarcted hearts, such as miR-1, miR-208 and miR-499, are carried in EVs and mobilise bone marrow progenitor cells ${ }^{66}$. Injured cardiomyocyte-derived EVs accelerate transplanted MSC injury in infarcted hearts ${ }^{67}$.

MiR-132, delivered by MSC-derived EVs, promotes angiogenesis in $\mathrm{MI}^{68}$. EVs derived from injured cardiomyocytes might impede the survival and promote the apoptosis of bone marrow-derived MSCs in the infarcted heart. Transplanted MSCs reduce autophagic flux in infarcted hearts via exosomal transfer of miR-125b ${ }^{69}$. cTnI-targeted EVs carrying miR-590-3p from MSCs were endocytosed by cardiomyocytes and thus promoted cardiomyocyte proliferation in the peri-MI area and eventually restored cardiac function as a treatment for $\mathrm{MI}^{70}$. Cell-free delivery of EVs secreted from iPSC-derived cardiomyocytes into the heart promotes heart recovery and treats myocardial injury ${ }^{71}$. EVs produced by cells under adverse conditions not only contribute to disease pathology but also might be detrimental towards cardiac regenerative therapies.

\section{Standardisation of EV studies}

Scientific studies have sharply increased in the past 10 years, analysing multiple functions of EVs in numerous physiological pathways, from ageing, obesity, CVDs to other diseases. EV research has now clearly gained widespread interest and enthusiasm. However, the promotion of rigorous EV research is an ongoing process due to a lack of well-accepted standards for performing EV studies, which hinders progress in studying the role of EVs as diagnostic biomarkers and therapeutic options for different diseases. The conclusions of several EV studies are not sufficiently supported by the experiments performed or the information reported. The
International Society for Extracellular Vesicles (ISEV) proposed the minimal information for studies of extracellular vesicles guideline in 2018 . The major goal of this guideline was to guide experimental requirements specific to the EV field and promote standardisation in the EV field. By improving the reliability and reproducibility of EV studies, EVs will have stronger potential as diagnostic biomarkers and therapeutic options for CVDs and other diseases.

\section{Conclusion}

Proper cardiovascular function depends on the coordinated interplay and communication between cardiomyocytes and noncardiomyocytes. Despite EVs representing a significant mechanism for intracellular communication, little is known about EV-mediated regulation of cardiomyocytes and noncardiomyocytes within the healthy and diseased heart. Moreover, how neighbouring or distant cells can secrete EVs to affect cardiac structure and function is largely unknown. On the one hand, miRs and proteins are transferred by EVs to maintain cardiovascular homeostasis, but on the other hand, the dysregulation of miRs and proteins transferred by EVs gives rise to the development of CVDs. Considering the fundamental roles of EVs in regulating physiological and pathological functions, future studies should be aimed at the broad and detailed understanding of EV functions and the development of new and personalised EV-based therapies.

\section{Acknowledgements \\ This work was supported by grants from the National Natural Science Foundation of China (81900357, 81903392 and 81941021), the Military Medical Science and Technology Youth Incubation Programme (20QNPY110), the National Key R\&D Programme of China (2016YFC0105006 and 2018YFC2000400), the National S\&T Resource Sharing Service Platform Project of China (YCZYPT[2018]07), the General Hospital of PLA Medical Big Data R\&D Project (MBD2018030), the China Postdoctoral Science Foundation Fund Project (2019M650359), the National Geriatric Disease Clinical Medicine Research Centre Project (NCRCG-PLAGH-2017-014), the Hainan Medical and Health Research Project (16A200057), the Sanya Medical and Health Science and Technology Innovation Project (2016YW21, 2017YW22 and 2018YW11), the Military Medicine Programme of the Chinese PLA General Hospital (QNF19069) and the Clinical Scientific Research Supporting Fund of the Chinese PLA General Hospital (2017FC-CXYY-3009).}

\footnotetext{
Author details

'Department of Geriatric Cardiology, Chinese People's Liberation Army General Hospital, Beijing 100853, China. ${ }^{2}$ Department of Cardiology, Hainan Hospital of Chinese People's Liberation Army General Hospital, Sanya 572013, China. ${ }^{3}$ Department of Epidemiology, School of Public Health, Southern Medical University, Guangzhou 510515, China. ${ }^{4}$ Central Laboratory, Hainan Hospital of Chinese People's Liberation Army General Hospital, Sanya 572013, China. ${ }^{5}$ Centre for the Study of Ageing and Human Development and Geriatrics Division, Medical School of Duke University, Durham, NC 27708, USA. ${ }^{6}$ Centre for Healthy Ageing and Development Studies, National School of Development, Peking University, Beijing 100871, China
}

Conflict of interest

The authors declare that they have no conflict of interest. 


\section{Publisher's note}

Springer Nature remains neutral with regard to jurisdictional claims in published maps and institutional affiliations.

Received: 18 May 2020 Revised: 25 June 2020 Accepted: 14 July 2020 Published online: 30 July 2020

\section{References}

1. Benjamin, E. J. et al. American Heart Association Statistics Committee and Stroke Statistics Subcommittee. Heart disease and stroke statistics-2017 update: a report from the American Heart Association. Circulation 135, e146-e603 (2017)

2. Gartz, M. \& Strande, J. L. Examining the paracrine effects of exosomes in cardiovascular disease and repair. J. Am. Heart Assoc. 7, pii: e007954 (2018).

3. Maia, J. et al. Exosome-based cell-cell communication in the tumor microenvironment. Front. Cell Dev. Biol. 6, 18 (2018).

4. Sahoo, S. \& Losordo, D. W. Exosomes and cardiac repair after myocardial infarction. Circ. Res. 114, 333-344 (2014)

5. Colombo, M., Raposo, G. \& Théry, C. Biogenesis, secretion, and intercellular interactions of exosomes and other extracellular vesicles. Annu. Rev. Cell Dev. Biol. 30, 255-289 (2014).

6. Pfeifer, P., Werner, N. \& Jansen, F. Role and function of microRNAs in extracellular vesicles in cardiovascular biology. Biomed. Res. Int. 2015, 161393 (2015).

7. Sarlon-Bartoli, G. et al. Plasmatic level of leukocyte-derived microparticles is associated with unstable plaque in asymptomatic patients with high-grade carotid stenosis. J. Am. Coll. Cardiol. 62, 1436-1441 (2013).

8. Rautou, P. E. et al. Microparticles, vascular function, and atherothrombosis. Circ. Res. 109, 593-606 (2011).

9. Diehl, P. et al. Microparticles: major transport vehicles for distinct microRNAs in circulation. Cardiovasc. Res. 93, 633-644 (2012).

10. Loyer, X. et al. Microvesicles as cell-cell messengers in cardiovascular diseases. Circ. Res. 114, 345-353 (2014).

11. Schober, A., Nazari-Jahantigh, M. \& Weber, C. microRNA mediated mechanisms of the cellular stress response in atherosclerosis. Nat. Rev. Cardiol. 13, 120 (2016).

12. Liu, Y. et al. Atherosclerotic conditions promote the packaging of functional microRNA-92a-3p into endothelial microvesicles.atherosclerotic conditions promote the packaging of functional microRNA-92a-3p into endothelial microvesicles. Circ. Res. 124, 575-587 (2019).

13. Li, H. et al. Coronary serum exosomes derived from patients with myocardial ischemia regulate angiogenesis through the miR-939-mediated nitric oxide signaling pathway. Theranostics 8, 2079-2093 (2018).

14. Miller, V. M. et al. Specific cell-derived microvesicles: linking endothelial function to carotid artery intima-media thickness in low cardiovascular risk menopausal women. Atherosclerosis 246, 21-28 (2016).

15. Holvoet, P. et al. Low MT-CO1 in monocytes and microvesicles is associated with outcome in patients with coronary artery disease. J. Am. Heart Assoc. 5, e004207 (2016).

16. Niu, C. et al. Macrophage foam cell-derived extracellular vesicles promote vascular smooth muscle cell migration and adhesion. J. Am. Heart Assoc. 5, e004099 (2016)

17. Jansen, F. et al. Endothelial microparticle-mediated transfer of microRNA-126 promotes vascular endothelial cell repair via spred1 and is abrogated in glucose-damaged endothelial microparticles. Circulation 128, 2026-2038 (2013).

18. Zernecke, A. et al. Delivery of microRNA-126 by apoptotic bodies induces CXCL12-dependent vascular protection. Sci. Signal. 2, ra81 (2009).

19. Jansen, F. et al. MicroRNA expression in circulating microvesicles predicts cardiovascular events in patients with coronary artery disease. J. Am. Heart Assoc. 3, e001249 (2014).

20. Rautou, P. E. et al. Microparticles from human atherosclerotic plaques promote endothelial ICAM-1-dependent monocyte adhesion and transendothelial migration. Circ. Res. 108, 335-343 (2011).

21. Caporali, A. et al. p75(NTR)-dependent activation of NF-kB regulates microRNA-503 transcription and pericyte-endothelial crosstalk in diabetes after limb ischaemia. Nat. Commun. 6, 8024 (2015).

22. Hergenreider, E. et al. Atheroprotective communication between endothelial cells and smooth muscle cells through miRNAs. Nat. Cell Biol. 14, 249-256 (2012).
23. Zhao, J. et al. Mesenchymal stromal cell-derived exosomes attenuate myocardial ischaemia-reperfusion injury through miR-182-regulated macrophage polarization. Cardiovasc. Res. 115, 1205-1216 (2019).

24. Zhu, L. P. et al. Hypoxia-elicited mesenchymal stem cell-derived exosomes facilitates cardiac repair through miR-125b-mediated prevention of cell death in myocardial infarction. Theranostics 8, 6163-6177 (2018).

25. Badrnya, S., Baumgartner, R. \& Assinger, A. Smoking alters circulating plasma microvesicle pattern and microRNA signatures. Thromb. Haemost. 112, 128-136 (2014).

26. Vicencio, J. M. et al. Plasma exosomes protect the myocardium from ischemiareperfusion injury. J. Am. Coll. Cardiol. 65, 1525-1536 (2015).

27. Ribeiro-Rodrigues, T. M. et al. Exosomes secreted by cardiomyocytes subjected to ischaemia promote cardiac angiogenesis. Cardiovasc Res. 113, 1338-1350 (2017).

28. Velez, P. et al. Identification of a circulating microvesicle protein network involved in ST-elevation myocardial infarction. Thromb. Haemost. 112, 716-726 (2014).

29. An, M. et al. Extracellular matrix-derived extracellular vesicles promote cardiomyocyte growth and electrical activity in engineered cardiac atria. Biomaterials 146, 49-59 (2017)

30. Qiao, L. et al. microRNA-21-5p dysregulation in exosomes derived from heart failure patients impairs regenerative potential. J. Clin. Investig. 129, 2237-2250 (2019).

31. Bang, C. et al. Cardiac fibroblast-derived microRNA passenger strand-enriched exosomes mediate cardiomyocyte hypertrophy. J. Clin. Investig. 124, 2136-2146 (2014)

32. Datta, R. et al. Myocyte-derived Hsp90 modulates collagen upregulation via biphasic activation of STAT-3 in fibroblasts during cardiac hypertrophy. Mol. Cell Biol. 37, e00611-e00616 (2017)

33. Wang, $X$. et al. Cardiomyocytes mediate anti-angiogenesis in type 2 diabetic rats through the exosomal transfer of miR-320 into endothelial cells. J. Mol. Cell Cardiol. 74, 139-150 (2014).

34. Halkein, J. et al. MicroRNA-146a is a therapeutic target and biomarker for peripartum cardiomyopathy. J. Clin. Investig. 123, 2143-2154 (2013).

35. Oh, J. G. et al. miR-146a suppresses SUMO1 expression and induces cardiac dysfunction in maladaptive hypertrophy. Circ. Res. 123, 673-685 (2018).

36. Wider, J. et al. Remote ischemic preconditioning fails to reduce infarct size in the Zucker fatty rat model of type-2 diabetes: role of defective humoral communication. Basic Res. Cardiol. 113, 16 (2018).

37. Muller, G. Microvesicles/exosomes as potential novel biomarkers of metabolic diseases. Diabetes Metab. Syndr. Obes. 5, 247-282 (2012).

38. Zhang, Y. et al. Adipocyte-derived microvesicles from obese mice induce M1 macrophage phenotype through secreted miR-155. J. Mol. Cell Biol. 8, 505-517 (2016).

39. Barberio, M. D. et al. Cholesterol efflux alterations inadolescent obesity: role of adipose-derived extracellular vesical microRNAs. J. Transl. Med. 17, 232 (2019)

40. Wang, F. et al. Insulin resistance adipocyte-derived exosomes aggravate atherosclerosis by increasing vasa vasorum angiogenesis in diabetic ApoE-/mice. Int. J. Cardiol. 265, 181-187 (2018).

41. Liu, J. et al. miR-93-5p-containing exosomes treatment attenuates acute myocardial infarction-induced myocardial damage. Mol. Ther. Nucleic Acids 11 103-115 (2018).

42. Xie, Z. et al. Adipose-derived exosomes exert proatherogenic effects by regulating macrophage foam cell formation and polarization. J. Am. Heart Assoc. 7, e007442 (2018)

43. Deng, Z. B. et al. Adipose tissue exosome-like vesicles mediate activation of macrophage-induced insulin resistance. Diabetes 58, 2498-2505 (2009).

44. Hou, Z. et al. Longterm exercise-derived exosomal miR-342-5p. Circ. Res. 124, 1386-1400 (2019).

45. Chen, W. K. et al. Exercise training augments Sirt1-signaling and attenuates cardiac inflammation in D-galactose induced-aging rats. Aging 10, 4166-4174 (2018).

46. Benedikter, B. J. et al. Proteomic analysis reveals procoagulant properties of cigarette smoke-induced extracellular vesicles. J. Extracell. Vesicles 8, 1585163 (2019).

47. Liu, X. et al. miRNA profiling of exosomes from spontaneous hypertensive rats using next-generation sequencing. Cardiovasc Transl. Res. 76, 1770 (2018).

48. Pironti, G. et al. Circulating exosomes induced by cardiac pressure overload contain functional angiotensin II type 1 receptors. Circulation $\mathbf{2 4 ,}$ 2120-2130 (2015). 
49. Davidson, S. M. et al. Cardioprotection mediated by exosomes is impaired in the setting of type II diabetes but can be rescued by the use of non-diabetic exosomes in vitro. J. Cell Mol. Med. 22, 141-151 (2018).

50. Freeman, D. W. et al. Altered extracellular vesicle concentration, cargo and function in Diabetes mellitus. Diabetes 67, 2377-2388 (2018).

51. Hu, J. et al. Exosomal Mst1 transfer from cardiac microvascular endothelial cells to cardiomyocytes deteriorates diabetic cardiomyopathy. Biochim. Biophys. Acta Mol. Basis Dis. 1864, 3639-3649 (2018).

52. Alvarez-Erviti, L. et al. Delivery of siRNA to the mouse brain by systemic injection of targeted exosomes. Nat. Biotechnol. 29, 341-345 (2011).

53. Tang, K. et al. Delivery of chemotherapeutic drugs in tumour cell-derived microparticles. Nat. Commun. 3, 1282 (2012).

54. Ha, D., Yang, N. \& Nadithe, V. Exosomes as therapeutic drug carriers and delivery vehicles across biological membranes: current perspectives and future challenges. Acta Pharm. Sin. B 4, 287-296 (2016)

55. Bohao, Liu et al. Cardiac recovery via extended cell-free delivery of extracellular vesicles secreted by cardiomyocytes derived from induced pluripotent stem cells. Nat. Biomed. Eng. 2, 293-303 (2018).

56. Song, $Y$. et al. Localized injection of miRNA-21-enriched extracellular vesicles effectively restores cardiac function after myocardial infarction. Theranostics $\mathbf{9}$, 2346-2360 (2019).

57. Wang, X. et al. Engineered exosomes with ischemic myocardium-targeting peptide for targeted therapy in myocardial infarction. J. Am. Heart Assoc. 7, e008737 (2018).

58. Liu, L. et al. Exosomes derived from mesenchymal stem cells rescue myocardial ischaemia/reperfusion injury by inducing cardiomyocyte autophagy via AMPK and Akt pathways. Cell Physiol. Biochem. 43, 52-68 (2017).

59. Mackie, A. R. et al. Sonic hedgehogmodified human CD34 + cells preserve cardiac function after acute myocardial infarction. Circ. Res. 111, 312-321 (2012).

60. Wang, Y. et al. Exosomes/microvesicles from induced pluripotent stem cells deliver cardioprotective miRNAs and prevent cardiomyocyte apoptosis in the ischemic myocardium. Int. J. Cardiol. 192, 61-69 (2015).
61. Gallet, R. et al. Exosomes secreted by cardiosphere-derived cells reduce scarring, attenuate adverse remodelling, and improve function in acute and chronic porcine myocardial infarction. Eur. Heart J. 38, 201-211 (2017).

62. $\mathrm{Yu}, \mathrm{B}$. et al. Exosomes secreted from GATA-4 overexpressing mesenchymal stem cells serve as a reservoir of anti-apoptotic microRNAs for cardioprotection. Int. J. Cardiol. 1, 349-360 (2015).

63. Bian, S. et al. Extracellular vesicles derived from human bone marrow mesenchymal stem cells promote angiogenesis in a rat myocardial infarction model. J. Mol. Med. 92, 387-397 (2014).

64. Lai, R. C. et al. Exosome secreted by MSC reduces myocardial ischemia/ reperfusion injury. Stem Cell Res. 4, 214-222 (2010).

65. Zhang, Z. et al. Pretreatment of cardiac stem cells with exosomes derived from mesenchymal stem cells enhances myocardial repair. J. Am. Heart Assoc. 5 e002856 (2016).

66. Cheng, M. et al. Circulating myocardial microRNAs from infarcted hearts are carried in exosomes and mobilise bone marrow progenitor cells. Nat. Commun. 10, 959 (2019)

67. Hu, M. et al. The harsh microenvironment in infarcted heart acceleratestransplanted bone marrow mesenchymal stem cells injury: the role of injuredcardiomyocytes-derived exosomes. Cell Death Dis. 9, 357 (2018).

68. Ma, T. et al. MicroRNA-132, delivered by mesenchymal stem cell-derived exosomes, promote angiogenesis in myocardial infarction. Stem Cells Int. 2018, 3290372 (2018).

69. Xiao, C. et al. Transplanted MSCs reduce autophagic flux in infarcted hearts via the exosomal transfer of miR-125b. Circ. Res. 123, 564-578 (2018).

70. Wang, Y. et al. Rapid delivery of Hsa-miR-590-3p using targeted exosomes to treat acute myocardial infarction through regulation of the cell cycle. $J$. Biomed. Nanotechnol. 14, 968-977 (2018).

71. Liu, B. et al. Cardiac recovery via extended cell-free delivery of extracellular vesicles secreted by cardiomyocytes derived from induced pluripotent stem cells. Nat. Biomed. Eng. 2, 293-303 (2018). 